\title{
Withdrawn notices
}

Bjermer L, Gauvreau G, Postma D, et al. P134 Methacholine challenge to demonstrate therapeutic equivalence of terbutaline via different turbuhaler devices in patients with mild to moderate asthma: appraisal of a phase iii, four-way crossover design. Thorax 2016;71:A155. doi:10.1136/thoraxjnl-2016-209333.277

This abstract has been withdrawn.

Thorax 2017;72:287. doi:10.1136/thoraxjnl-2016-209333.277wthn 